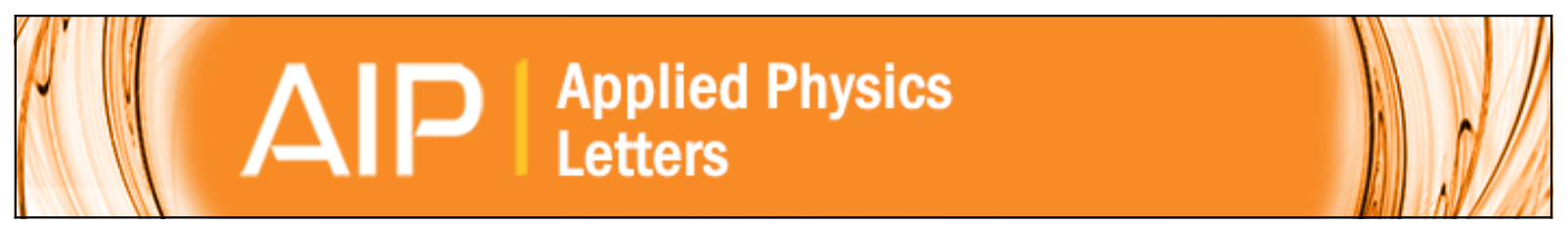

Dual-mode lasing in a 1310-nm quantum dot distributed feedback laser induced by single-beam optical injection

A. Hurtado, I. D. Henning, M. J. Adams, and L. F. Lester

Citation: Applied Physics Letters 102, 201117 (2013); doi: 10.1063/1.4807759

View online: http://dx.doi.org/10.1063/1.4807759

View Table of Contents: http://scitation.aip.org/content/aip/journal/apl/102/20?ver=pdfcov

Published by the AIP Publishing

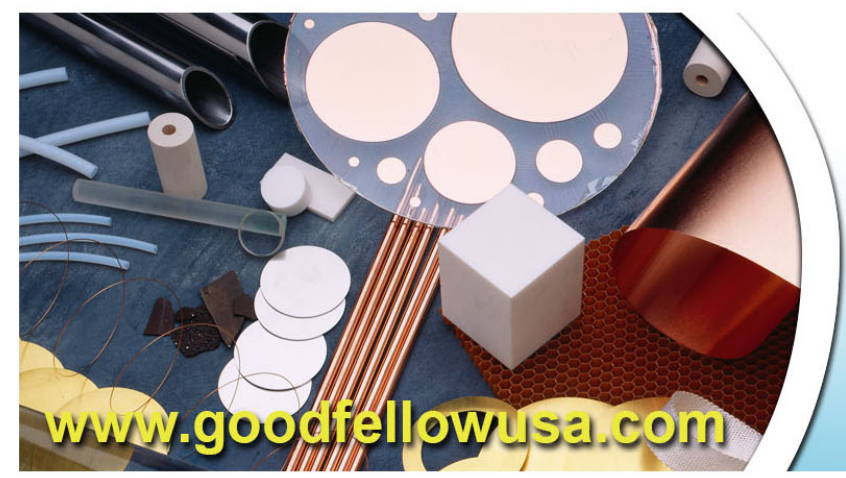

BOAdFEl|OII metals • ceramics $\cdot$ polymers composites $\bullet$ compounds • glasses

Save $5 \%$ B Buy online 70,000 products $\cdot$ Fast shipping 


\title{
Dual-mode lasing in a 1310-nm quantum dot distributed feedback laser induced by single-beam optical injection
}

\author{
A. Hurtado, ${ }^{1,2, a)}$ I. D. Henning, ${ }^{2}$ M. J. Adams, ${ }^{2}$ and L. F. Lester ${ }^{1}$ \\ ${ }^{1}$ Center for High Technology Materials, University of New Mexico, 1313 Goddard St. SE, Albuquerque, \\ New Mexico 87106, USA \\ ${ }^{2}$ School of Computer Science and Electronic Engineering, University of Essex, Wivenhoe Park, \\ Colchester CO4 3SQ, United Kingdom
}

(Received 1 March 2013; accepted 11 May 2013; published online 24 May 2013)

\begin{abstract}
Tunable dual-mode lasing is experimentally demonstrated in a 1310-nm quantum dot (QD) distributedfeedback (DFB) laser under single-beam optical injection. The wavelength spacing between the two lasing modes is controlled by injecting the external optical signal into different residual Fabry-Perot modes of the QD DFB laser. The influence of important parameters, i.e., injection strength and bias current, is also analyzed. The simple experimental configuration used to achieve tunable dual-mode lasing and the theoretically superior properties of the QD laser offer exciting prospects for the use of these devices in microwave signal generation and radio-over-fiber applications for future mobile communication networks. C 2013 AIP Publishing LLC. [http://dx.doi.org/10.1063/1.4807759]
\end{abstract}

Quantum dot (QD) lasers possess particular physical characteristics that make them attractive for stable optical injection from an external source, particularly a small linewidth enhancement factor and a strong modulation damping rate. ${ }^{1-3}$ Therefore, optically injected QD lasers have recently received considerable attention (see Ref. 4, and references therein). However, in spite of these interesting features, the effect of optical injection in assisting dual-mode lasing in a QD laser has not been yet analyzed. For dual-mode laser operation, the significant inhomogeneous broadening of the QD gain medium is advantageous. ${ }^{5}$ Although dual mode lasing has been previously studied in a QD device, the effect of optical injection in assisting this behavior has not been examined. Previous dual-mode QD lasers consisted of a split-anode geometry that enabled asymmetric pumping to balance the emission between one mode that was coupled to a Bragg grating and the other to the Fabry-Perot (FP) cavity. ${ }^{5,6}$ Simultaneous ground state-excited state and excited state-excited state emission were demonstrated with mode frequency differences varying from 1.3 to $8 \mathrm{THz} .^{5}$ Optical feedback and temperature control were identified as additional techniques for tuning the frequency difference. ${ }^{6}$ These results were enabled through the unique nature of the QD media. In this work, we report dual-mode lasing operation in a QD distributed-feedback (DFB) laser induced by single-beam optical injection into one of the residual FP modes of the device. Tunability of the dual-mode lasing is also demonstrated by injecting external light into different FP modes of the QD DFB laser.

In this work, we have used a 1310-nm laterally loss coupled 300- $\mu \mathrm{m}$ long DFB QD laser with asymmetrically HR-HR (high reflectivity) coated facets having front and back facet reflectivity equal to $80 \%$ and $95 \%$. A chromium grating with $200-\mathrm{nm}$ pitch provided distributed reflectivity. This structure facilitates single-mode emission and suppresses FP cavity modes. The epitaxial layer structure of the device is shown in

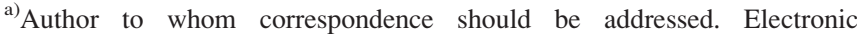
addresses: ahurta01@unm.edu and ahurt@essex.ac.uk
}

Fig. 1(a). The active region consists of six $9.1 \mathrm{~nm}$-thick InAs/ InGaAs dots-in-a-well (DWELL) layers separated by 30-nm GaAs barriers. More details on the device structure can be found in Refs. 7 and 8. Fig. 1(b) shows the LI curve of the device measured at $298 \mathrm{~K}$ exhibiting a threshold current of about 4.4 mA. Optical spectra of the QD DFB laser for different applied bias currents are shown in Figs. 1(c) and 1(d). Specifically, in Fig. 1(c), a current of $4.6 \mathrm{~mA}$ is applied whereas currents ranging from 6 to $30 \mathrm{~mA}$ were applied for the spectra in Fig. 1(d). The QD laser has an emission wavelength between $1309 \mathrm{~nm}$ and $1310 \mathrm{~nm}$ depending on the biasing conditions. Additional attenuated FP modes as well as the main lasing mode are clearly seen in Fig. 1(c). The FP modes are separated by approximately $0.8 \mathrm{~nm}$, and a measured side mode suppression ratio (SMSR) in excess of $35 \mathrm{~dB}$ is observed. Throughout this work, we will refer, respectively, to the main lasing and to the subsidiary attenuated modes of the QD DFB laser as Bragg mode $\left(\lambda_{\text {Bragg }}\right)$ and residual FP modes ( $\left.\lambda_{\text {FPmode }}\right)$.

Fig. 2 shows the experimental setup used to demonstrate optical-injection induced dual-mode operation in a QD DFB laser. Light from a 1312-nm Quantum Well (QW) DFB laser (Master Laser, ML) is injected into the 1310-nm QD DFB laser (Slave Laser, SL). The temperature of the SL is kept constant at $298 \mathrm{~K}$ throughout the experiments. An optical isolator is included to avoid back-reflections into the ML. A polarization controller and a variable optical attenuator (VOA) are used to control the polarization and input power of the ML's light. The polarization of the external signal is set to align with that of the SL. A 90/10 fiber coupler divides the external signal into two paths. The $10 \%$ port is used to monitor the optical input power with a power meter, whereas the $90 \%$ port is injected into the SL via a polarization maintaining circulator. Finally, the light reflected by the SL is collected and analyzed using an Optical Spectrum Analyzer (OSA).

Traditionally, in the analysis of optically injected semiconductor lasers (either planar or vertical-cavity devices, with bulk or QW active regions), when light is injected into one of the SL's subsidiary modes and the injection is 
(a)

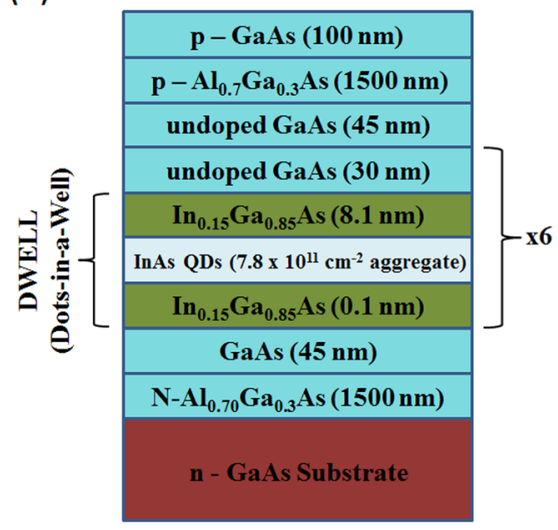

(b)

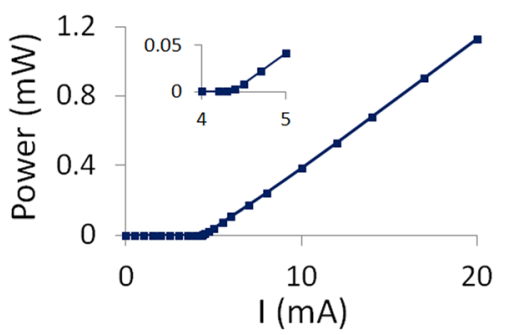

(c)

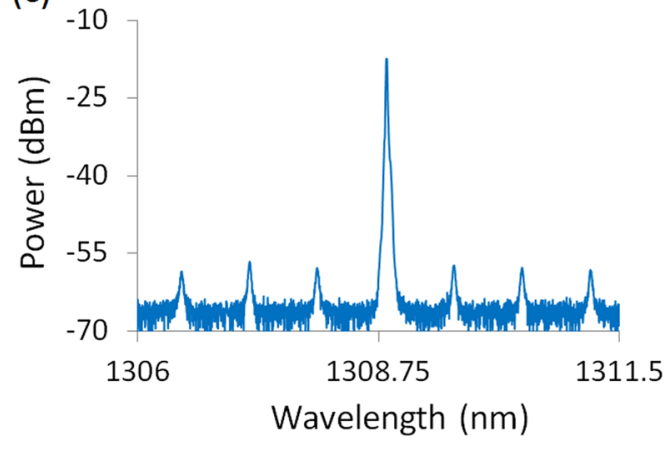

(d)

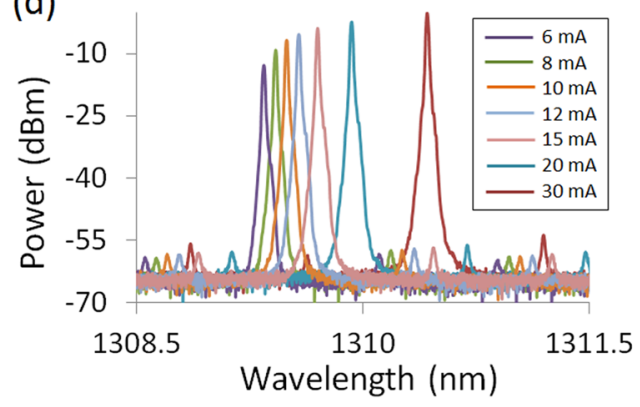

FIG. 1. (a) Epitaxial layer structure (b) LI curve at $298 \mathrm{~K}$, and (c) and (d) optical spectra of the $1310 \mathrm{~nm}$ QD DFB laser when biased with (c) $4.6 \mathrm{~mA}$ and (d) from 6 to $30 \mathrm{~mA}$ (also at $298 \mathrm{~K}$ ). sufficiently strong, the latter locks to the injected light's wavelength. Simultaneously, the SL's lasing mode(s) is(are) suppressed or very strongly attenuated. Thus, after the injection locking process, single-mode lasing is achieved at the SL's output. This mechanism has been utilized in several applications, such as optical logic, optical switching/routing, and wavelength channel selection in optical access networks (for a review, see Ref. 9, and references therein).

An analogous response is observed for the QD DFB laser of this work when the device is biased with currents close to threshold. Fig. 3(a) shows optical spectra of the solitary SL (red dashed line) and after the injection of the ML's light into one of the residual FP modes (blue solid line). The device was biased with $8 \mathrm{~mA}\left(1.81 \times \mathrm{I}_{\mathrm{th}}\right)$, the ML's optical power was set at $4.8 \mathrm{~mW}$, and an initial frequency detuning of $\Delta \mathrm{f}=-4 \mathrm{GHz}$ was set between the resonant frequencies of the $\mathrm{ML}$ and the residual FP mode of the QD DFB laser $\left(\Delta \mathrm{f}=\mathrm{f}_{\mathrm{ML}}-\mathrm{f}_{\mathrm{FPmode}}\right)$. Fig. 3(a) shows that after the ML's injection, locking of the SL's residual mode to the external signal with simultaneous suppression of the device's Bragg mode is produced. Thus, single-mode laser operation with emission at the ML's wavelength is obtained (with a SMSR over $50 \mathrm{~dB}$ ). Figs. 3(b) and 3(c) plot in more detail both the

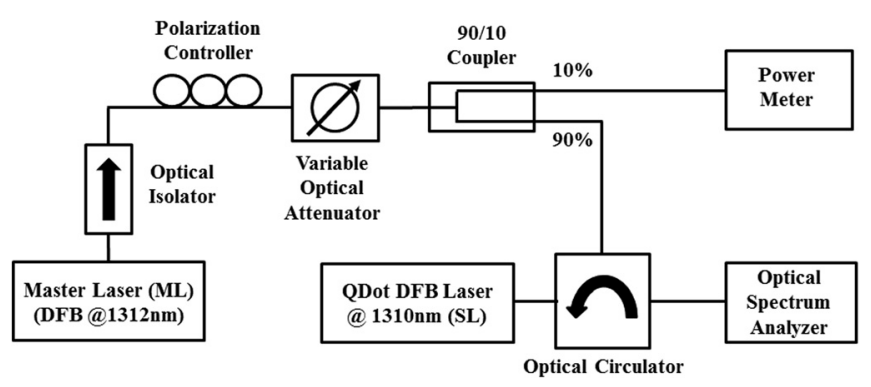

FIG. 2. Experimental setup.
Bragg and injected residual FP modes for the same conditions as in Fig. 3(a) and for the case in which the injected optical power is increased from 0 to $4.8 \mathrm{~mW}$. As seen in Figs. 3(b) and 3(c), increasing the injection strength reduces the Bragg mode's power, shifting its emission towards longer wavelengths. This process, which is initially modest, is enhanced after a particular threshold in injected power above which the Bragg mode suffers a drastic attenuation and its wavelength is further shifted to the longer wavelength side. Simultaneously, the residual FP mode locks to the ML's wavelength (detuned to its longer wavelength side, as seen in Fig. 3(c)). Further increasing the injection strength does not change the induced single-mode lasing response at the ML's wavelength.

On the other hand, a different response is observed when the QD DFB laser is biased with a higher current well above threshold. Fig. 4 plots results with the device biased with $30 \mathrm{~mA}\left(6.81 \times \mathrm{I}_{\mathrm{th}}\right)$. Fig. 4(a) plots optical spectra at the output of the QD laser when operating in free-running mode (red dashed line) and after the injection of an external signal with $4.8 \mathrm{~mW}$ of optical power and an initial frequency detuning of $\Delta \mathrm{f}=-4 \mathrm{GHz}$ (blue solid line). Fig. 4(a) shows that as in the lower bias case of Fig. 3(a), the residual FP mode locks to the ML's signal following external injection; however, for a higher bias current after injection locking has been produced, the SL's Bragg mode is only slightly attenuated. To better observe this response, Figs. 4(b) and 4(c) plot the evolution of both the Bragg and the residual FP modes of the SL for increasing injection strength from 0 to $4.8 \mathrm{~mW}$. Initially, Fig. 4(b) shows a similar evolution as that observed in the lower bias case (depicted in Fig. 3(b)) with the Bragg mode experiencing a modest power reduction and reduced wavelength pull towards longer wavelengths. After a threshold in injection power is exceeded, a drastic pull in wavelength is observed with an associated power drop. 

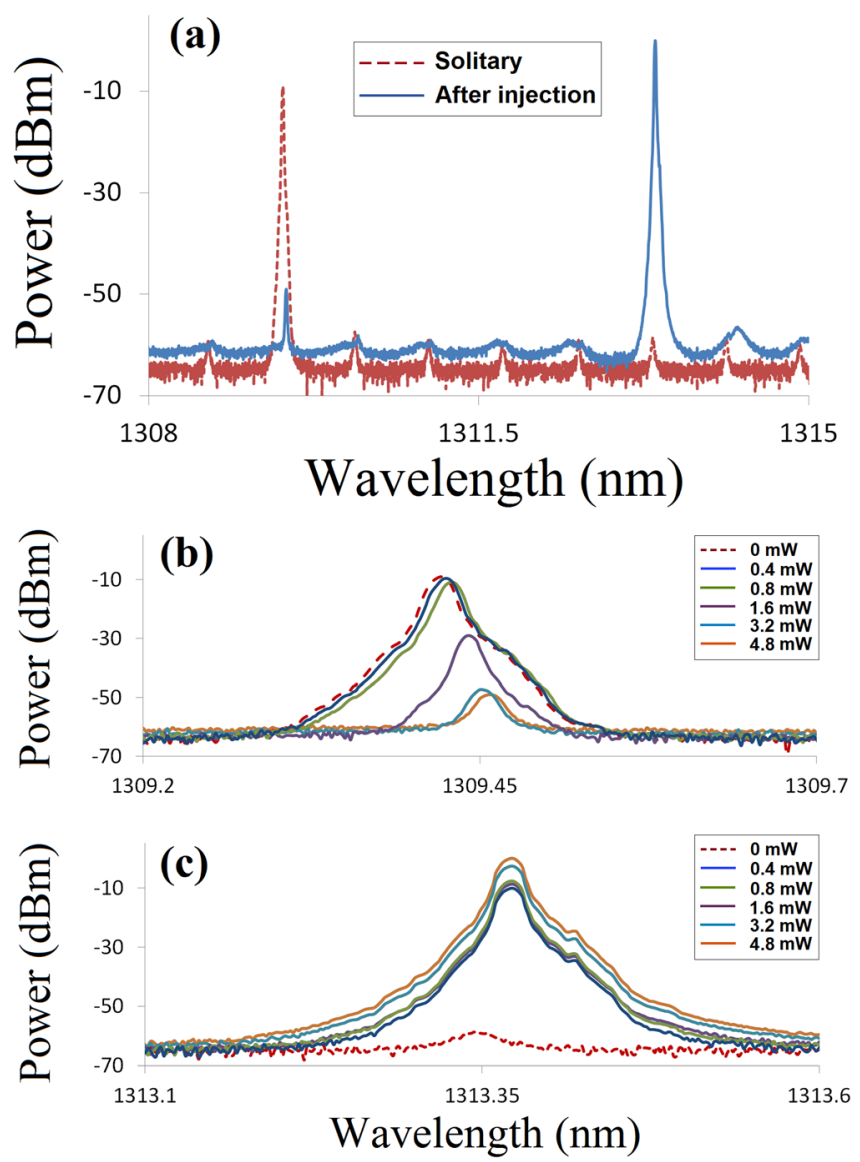

FIG. 3. (a) Spectra of the QD DFB laser in solitary (red dashed line) and after optical injection into the fifth residual FP mode in the longer wavelength side of the Bragg mode with $\mathrm{P}_{\text {inj }}=6.4 \mathrm{~mW} ; \Delta \mathrm{f}=-4 \mathrm{GHz}$ (blue solid line). (b) and (c) Spectra of the Bragg mode (b) and injected residual FP mode (c) of the device when the optical power of the injected signal is increased from 0 to $4.8 \mathrm{~mW}$. $\mathrm{I}_{\text {Bias }}=8 \mathrm{~mA}$.

Nevertheless, such a drop in power though abrupt is small $(\sim 1.35 \mathrm{~dB})$ and does not yield the total suppression of the lasing mode. Therefore, after locking both modes, the Bragg and the residual FP mode co-exist simultaneously. As a consequence, dual-mode lasing operation is induced at the output of the QD DFB laser by means of the single-beam optical injection mechanism. Further increases of injection strength do not change this status and dual-mode operation is achieved for all powers above the locking point.

Fig. 5 plots superimposed optical spectra of the QD DFB laser for different bias currents from $6 \mathrm{~mA}$ to $30 \mathrm{~mA}$, when subject to external optical injection into the fifth residual FP mode (on the longer wavelength side of the lasing mode) with $\mathrm{P}_{\mathrm{inj}}=4.8 \mathrm{~mW}$ and $\Delta \mathrm{f}=-4 \mathrm{GHz}$. In all cases plotted in Fig. 5, injection locking to the ML's wavelength has already been produced. However, it is only at the lower bias currents ( 6 and $8 \mathrm{~mA})$ that the Bragg mode is suppressed after the locking transition. As seen in Fig. 5, above approximately $10 \mathrm{~mA}$, the Bragg mode cannot be suppressed after locking, which yields dual-mode operation for currents exceeding this value. This is illustrated in the inset within Fig. 5 which plots the peak powers of both the residual FP (green) and Bragg modes (red) of the QD DFB laser as a function of applied bias. The separation between the two lines in the inset corresponds directly to the suppression ratio
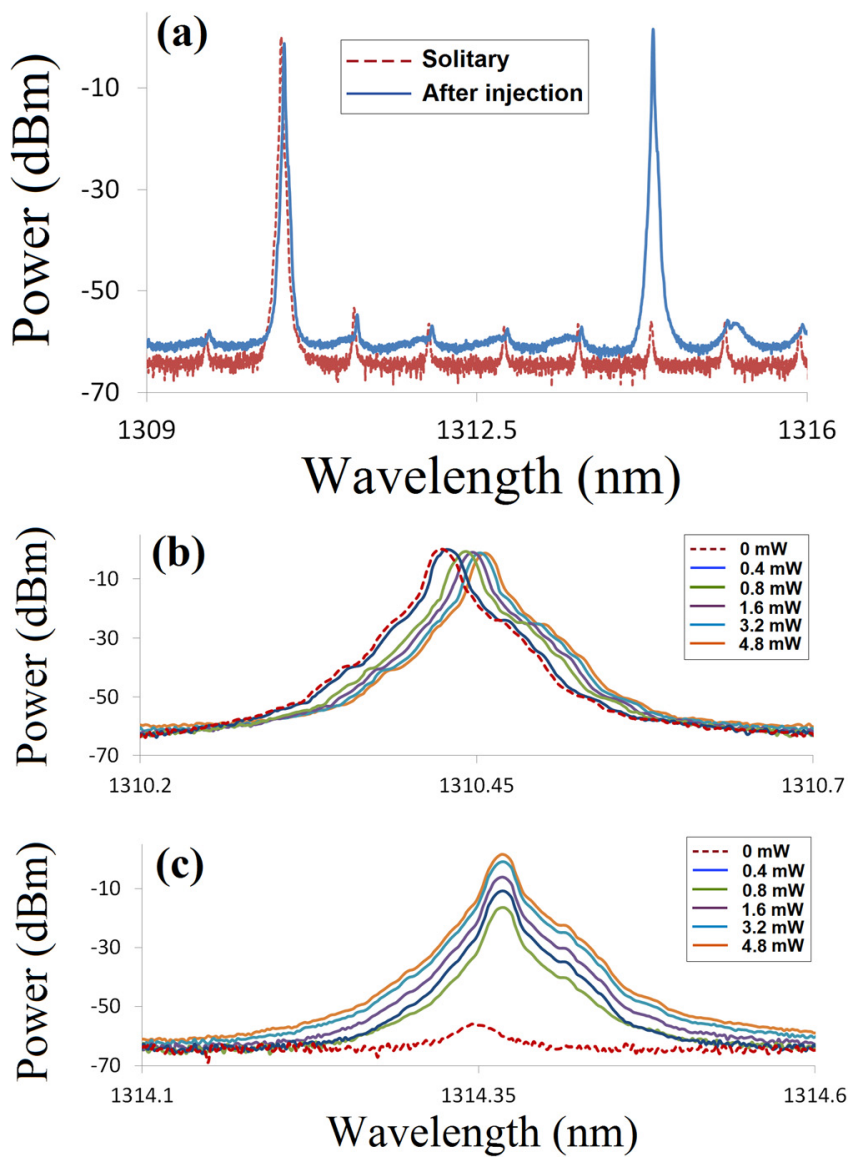

FIG. 4. (a) Spectra of the QD DFB laser in solitary (red dashed line) and after optical injection into the fifth residual FP mode on the longer wavelength side of the Bragg mode with $\mathrm{P}_{\mathrm{inj}}=4.8 \mathrm{~mW} ; \Delta \mathrm{f}=-4 \mathrm{GHz}$ (blue solid line). (b) and (c) Spectra of the Bragg (b) and injected residual FP mode (c) of the device when the optical power of the injected signal is increased from 0 to $4.8 \mathrm{~mW}$. $\mathrm{I}_{\text {Bias }}=30 \mathrm{~mA}$.

between the two modes, which clearly reduces for currents over $10 \mathrm{~mA}$.

Experiments have also been carried out in which the optical injection is assessed for other residual FP modes to show

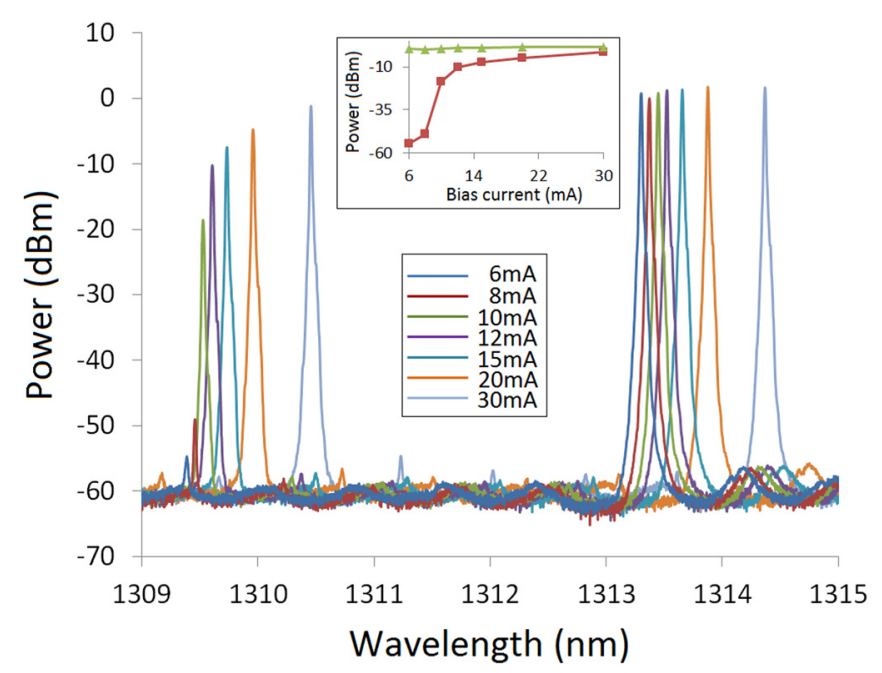

FIG. 5. Superimposed spectra of the QD DFB laser after the injection of an optical signal with $P_{\text {inj }}=4.8 \mathrm{~mW}$ and $\Delta \mathrm{f}=-4 \mathrm{GHz}$ into the fifth residual FP mode of the device (on the Bragg mode's longer wavelength side). Bias currents from 6 to $30 \mathrm{~mA}$ are applied. The inset plots the peak powers of the residual FP (green) and the Bragg mode (red) of the QD DFB laser vs. applied bias current. 

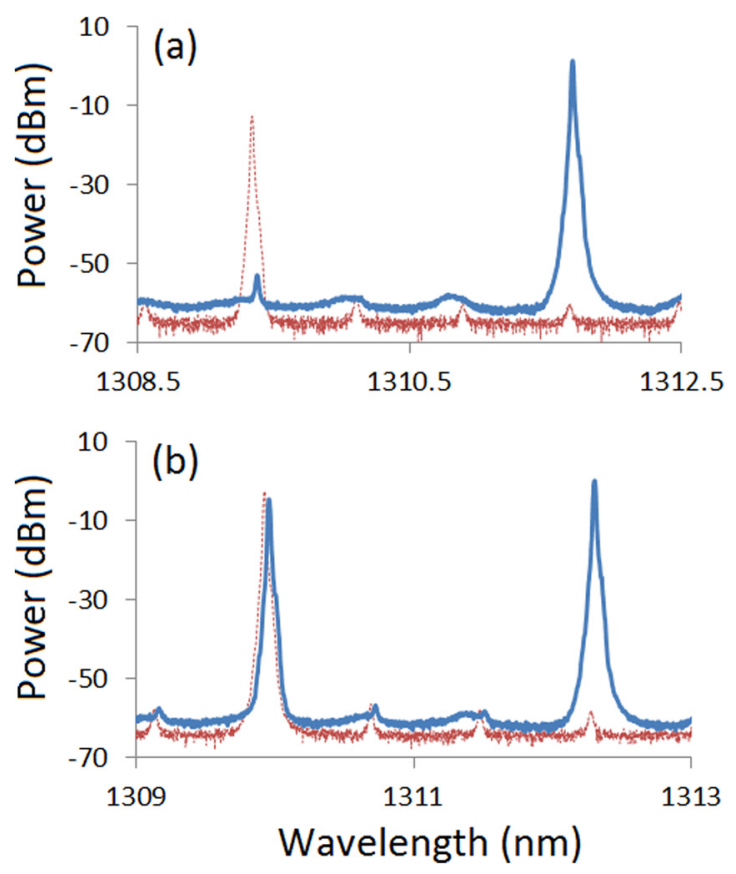

FIG. 6. Spectra of the QD DFB laser in solitary (red dashed lines) and after optical injection into the third residual FP mode in the longer wavelength side of the Bragg mode with $\mathrm{P}_{\text {inj }}=4.8 \mathrm{~mW} ; \Delta \mathrm{f}=-4 \mathrm{GHz}$ (blue solid lines). (a) $\mathrm{I}_{\text {Bias }}=6 \mathrm{~mA}$ and (b) $\mathrm{I}_{\text {Bias }}=20 \mathrm{~mA}$.

that dual-mode lasing is not limited to a single configuration of the device. Fig. 6 shows similar response to that described in Figs. 3 and 4 with the ML's light injected into the third residual FP mode on the longer wavelength side of the Bragg line. The same initial conditions as in Figs. 3(a) and 4(a) are applied for the results plotted in Figs. 6(a) and 6(b). Two bias currents equal to $6 \mathrm{~mA}\left(1.36 \times \mathrm{I}_{\mathrm{th}}\right)$ and $20 \mathrm{~mA}\left(4.54 \times \mathrm{I}_{\mathrm{th}}\right)$ were configured, respectively, for the cases depicted in Figs. 6(a) and 6(b). The injection power was in both cases high enough to produce the locking of the residual FP mode to the external signal. Once again, Fig. 6(a) shows that the Bragg mode is suppressed after locking at lower bias, whereas Fig. 6(b) illustrates that higher bias supports the Bragg mode and enables dual-mode lasing. Therefore, simply by injecting the external light into different residual FP modes of the SL, the wavelength separation between the two lasing modes can be tuned. The potential for tunability of the dual-mode lasing operation offers exciting prospects for the use of nanostructure lasers as high frequency sources for future mobile communications and radio-over-fiber systems since the frequency of the generated signals corresponds to the separation between the two lasing lines. ${ }^{10}$

It is believed that the optical-injection induced dualmode lasing response in the QD DFB laser is due to the interplay of homogeneous broadening and imperfect carrier clamping in the QD material. ${ }^{11}$ Initially for low enough values of bias current, the injection of the ML's light into the SL's residual FP mode draws gain from the Bragg mode. Ultimately, this effect leads to the suppression of the latter after a particular value of injection strength is exceeded, as is observed in Fig. 3(b). This is the action of the homogeneous broadening. However, imperfect carrier density clamping at threshold yields an unclamped gain spectrum above threshold. This in turn means that higher bias currents yield higher available gain in optical transitions away from the dominant lasing mode. Consequently, exceeding a certain applied bias current, which for the case of the QD DFB laser of this work corresponds to approximately $10 \mathrm{~mA}$, the gain in the Bragg mode is restored due to the lack of clamping, and the external optical injection is not capable to completely suppress the free-running emission of the QD laser.

In conclusion, dual-mode lasing has been experimentally demonstrated with a nanostructure laser by means of single-beam optical injection. Specifically, in this work we have used a $1310 \mathrm{~nm}$ QD DFB laser subject to optical injection into one of its residual FP modes. For high enough levels of applied bias current, single-beam injection is sufficient to yield dual-mode lasing due to the lack of suppression of the device's Bragg mode. We believe that such process is a consequence of the interplay between homogeneous broadening and the lack of carrier density clamping in the QD material. Moreover, the described dual-mode lasing system allows tunability of the wavelength spacing between the two emitting modes by injecting the external signal into different residual FP modes. These exciting results offer promise for the use of this coupled laser system for microwave signal generation for radio-over-fiber applications and future mobile communication systems.

This work has been funded in part by the European Commission under the Programme FP7 Marie Curie International Outgoing Fellowships (IOF) Grant No. PIOFGA-2010-273822 and the Air Force Office of Scientific Research Grant No. FA9550-10-1-0276 and AFRL Loan Agreement 2011003RY.

${ }^{1}$ T. C. Newell, D. Bossert, A. Stintz, B. Fuchs, K. J. Malloy, and L. F. Lester, IEEE Photon. Technol. Lett. 11, 1527 (1999).

${ }^{2}$ P. Bhattacharya, D. Klotzkin, O. Qasaimeh, W. Zhou, S. Krishna, and D. Zhu, IEEE J. Sel. Top. Quantum Electron. 6, 426 (2000).

${ }^{3}$ H. Su and L. F. Lester, J. Phys. D: Appl. Phys. 38, 2112 (2005).

${ }^{4}$ B. Kelleher, D. Goulding, S. P. Hegarty, G. Huyet, E. A. Viktorov, and T. Erneux, Optically Injected Single-Mode Quantum Dot Lasers in Quantum Dot Devices, edited by Z. M. Wang (Springer, NY, 2012), Chap. 1, pp. $1-22$.

${ }^{5}$ N. A. Naderi, F. Grillot, K. Yang, J. B. Wright, A. Gin, and L. F. Lester, Opt. Express 18, 27028 (2010).

${ }^{6}$ F. Grillot, N. A. Naderi, J. B. Wright, R. Raghunathan, M. T. Crowley, and L. F. Lester, Appl. Phys. Lett. 99, 231110 (2011).

${ }^{7}$ H. Su, L. Zhang, A. L. Gray, R. Wang, T. C. Newell, K. J. Malloy, and L. F. Lester, IEEE Photon. Technol. Lett. 15, 1504 (2003).

${ }^{8}$ L. Zhang, R. Wang, Z. Zou, A. Gray, L. Olana, T. Newell, D. Webb, P. Varangis, and L. F. Lester, Optical Fiber Communication Conference, Technical Digest, Paper No. FG2, Atlanta, GA (2003).

${ }^{9}$ M. J. Adams, A. Hurtado, D. Labukhin, and I. D. Henning, Chaos 20, 037102 (2010).

${ }^{10}$ X. Q. Qi and J. M. Liu, IEEE J. Sel. Top. Quantum Electron. 17, 1198 (2011).

${ }^{11}$ A. Markus, J. X. Chen, C. Paranthoen, A. Fiore, C. Platz, and O. GauthierLafaye, Appl. Phys. Lett. 82, 1818 (2003). 